\title{
Choroidal metastasis presenting with tumour surface orange lipofuscin pigment.
}

\author{
Amer N Masalha ${ }^{1 *}$, Ido D Fabian ${ }^{2,3 *}$, Victoria ML Cohen ${ }^{2,4}$, Mandeep S Sagoo ${ }^{2,4}$ \\ ${ }^{1}$ Department of Medicine, Tel-Aviv University, Israel \\ ${ }^{2}$ Department of Ocular Oncology, Goldschleger Eye Institute, Sheba Medical Center, Tel-Aviv University, Tel Aviv, \\ Israel \\ ${ }^{3}$ Department of Ocular Oncology, Moorfields Eye Hospital, London, UK \\ ${ }^{4}$ NIHR Biomedical Research Centre for Ophthalmology, University College London Institute of Ophthalmology, \\ Moorfields Eye Hospital, London, UK \\ ${ }^{*}$ These authors have contributed equally
}

\begin{abstract}
Background and Objective: In ocular oncology, orange-color lipofuscin is commonly associated with choroidal melanoma. We herein report a case series of choroidal metastases with overlying lipofuscin pigment.

Methods: A retrospective case series of 3 consecutive patients with choroidal metastasis with overlying orange-color pigment.

Results and Discussion: Three women who presented to the London Ocular Oncology Service with suspicious choroidal lesions were included in this study. Median age of presentation was 59 years old (mean: 58). On clinical examination, visual acuity of the affected eyes was 6/24 in 2 cases and counting fingers in one. All lesions were pale and showed subretinal fluid, and orange color lipofuscin overlying the tumours, which was also demonstrated as speckled hyperflourescence on autofluorescence. At presentation to our service, $2 / 3$ patients were already diagnosed with metastatic disease from primary breast and lung cancer, and one had no known extraocular malignancy, but was diagnosed with metastatic lung cancer following systemic workup.

Conclusion: Findings of this study suggest that orange lipofuscin pigment, although commonly associated with choroidal melanoma, can also be found overlying choroidal metastasis. In case both entities are considered part of the differential diagnosis, presence of orange pigment should be carefully interpreted and further ocular and extraocular evaluation is advised.
\end{abstract}

Keywords: Choroidal metastasis, Choroidal melanoma, Lipofuscin, Orange pigment

Accepted on March 29, 2019

\section{Introduction}

Lipofuscin is a brown-yellow polymeric autofluorescent material, a product of intracellular components' degradation that originates from autophagocytosis and found inside lysosomes. Lipofuscin cannot be degraded, nor exocytosed, and hence it continuously accumulates over time within postmitotic cells, such as neurons, cardiac myocytes and skeletal muscle fibers [1]. The deposition of lipofuscin decreases cellular adaptability and promotes the development of agerelated pathologies, such as neuro-degenerative diseases and heart failure. In addition, its accumulation is associated with other pathological conditions, including lysosomal storage diseases, radiation-induced injuries and tumors [2].

In the eye, lipofuscin is found in the retinal pigment epithelium (RPE), originating from incompletely digested photoreceptor outer segments. Its accumulation is associated with RPE cell dysfunction and age-related macular degeneration [3]. In the context of ocular oncology, orange-colored lipofuscin was found to be a risk factor for choroidal naevus growth and considered a hallmark for malignant transformation into melanoma [4-8].

Differentiating clinically between choroidal metastasis and melanoma, the most common intraocular malignancy and most common primary intraocular malignancy in adults [9-11], respectively, is not always straightforward, especially when there's no known primary extraocular cancer site. Intraocular tumor biopsy, while commonly used for prognostication, is rarely used for diagnosis. Lipofuscin in this context, if present, overlying the choroidal tumor, usually tilts the scales toward primary choroidal melanoma. Herein we report a case series of 3 patients that presented with choroidal metastasis, which featured overlying orange-colored lipofuscin, implying that this clinical sign should be cautiously interpreted in the context of differentiating between choroidal melanoma and metastasis. 


\section{Methods and Materials}

This was a retrospective chart review of consecutive cases of choroidal metastasis with overlying orange pigment that presented to the London Ocular Oncology Service from July, 2015 to August, 2017. The study was approved by the Moorfields Eye Hospital institutional review board in accordance with the tenets of the Declaration of Helsinki.

Data retrieved from medical records included age at presentation, gender, past medical history, smoking history, presenting signs, clinical data of first examination, including all imaging testing, treatments given, and follow-up clinical data until last examination.

\section{Case Series}

\section{Case 1}

A 54-year old white female smoker, with no previous adverse medical history, was referred to The London Ocular Oncology Service in September 2015 due to complaints of blurred vision in her left eye for several weeks and a suspicious choroidal lesion in the same eye. On examination, best corrected visual acuity (BCVA) was $6 / 6$ in the right eye and $6 / 24$ in the left eye. Both anterior segments and right fundus examination were normal. On left fundoscopy (Figure 1A), a pale choroidal lesion, located supero-temporal to the optic disc was noted, with overlying subretinal fluid and orange pigment. The latter was also demonstrated on $\mathrm{AF}$ in the form of speckled hyperfluorescense and on MultiColor Scanning Laser Imaging (Heidelberg Engineering Inc., Hemel Hempstead, UK; Figures $1 \mathrm{~B}$ and $1 \mathrm{C}$, respectively). The left macula was detached (Figure 1D). B scan ultrasonography found the lesion to be 6.7 $\mathrm{mm}$ in diameter and $2.3 \mathrm{~mm}$ in elevation and to have high internal echogenicity (Figure 1E) and colour flow mapping demonstrated an internal blood flow of $11 \mathrm{~cm} / \mathrm{sec}$. FA failed to show disk leakage, vasculitis or cystoid macular oedema, with speckled hyperfluorescence and staining of the lesion. (Figures 1F) ICG angiography was hypo-fluorescent in the area of the lesion (Figures $1 \mathrm{G}$ ), without any other subclinical lesions. The differential diagnosis included a primary amelanotic choroidal melanoma versus secondary intraocular spread from an unknown primary cancer. PET-CT scan revealed mediastinal, lung, adrenal and retroperitoneal lymph node disease with a large solid pelvic mass. Brain metastases were found on MRI. Further evaluation, including lung biopsy, diagnosed metastatic adenocarcinoma of the lung. She was treated with eye and whole brain irradiation, 30 Gy in 10 fractions, and systemic chemotherapy, but died 4 months after presentation.

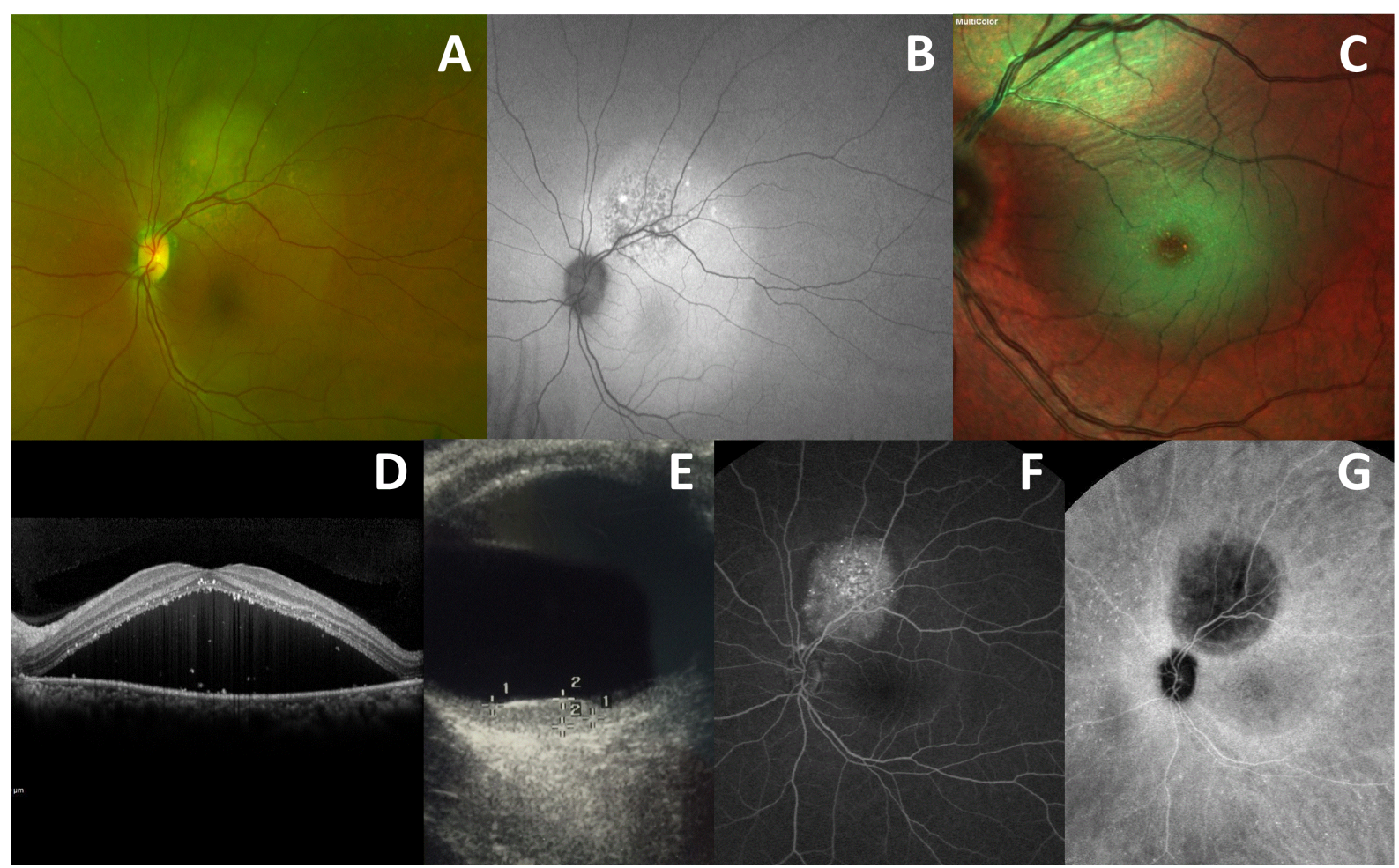

Figure 1. (A) Case 1 showing an amelanotic choroidal lesion superotemporal to the optic disc with overlying orange pigment, as shown on color fundus image, (B) on autofluorescence the lipofuscin is seen as speckled hyperfluorescent dots, (C) orange on multicolor scanning laser imaging, (D) subfoveal fluid as demonstrated on OCT, (E) ultrasound demonstrating the choroidal tumour, (F) Fluorescein angiography failed to show disk leakage, vasculitis or cystoid macular oedema, $(G)$ and there was masking on indocyanine green angiography.

\section{Case 2}

A 62-year old white woman, diagnosed with stage 4 metastatic breast cancer, was referred in August 2016 with complaints of flashing lights, deteriorated vision and a suspicious choroidal mass in the left eye. In 2011, she was diagnosed with triplenegative breast cancer, for which she underwent a lumpectomy, and adjuvant radiotherapy and chemotherapy. In 2013, she had a local recurrence, which was treated with mastectomy and further courses of systemic chemotherapy, and in 2016, she 
Citation: Masalha AN, Fabian ID, Cohen VML, et al. Choroidal metastasis presenting with tumour surface orange lipofuscin pigment. J Clin

Ophthalmol 2019;3(1):132-136.

was diagnosed with metastatic disease, including involvement of the lungs, bones and liver, for which she received various chemotherapeutic combinations, including fluorouracilepirubicin-cyclophosphamide (FEC) and docetaxelgemcitabine-carboplatin (GemCarbo). On examination, BCVA was $6 / 5$ in the right eye, with a normal examination and counting fingers in the left eye. She was pseudophakic in both eyes. On left fundoscopy, two choroidal deposits were seen, one large lesion abutting the infero-temporal aspect of the optic disk, extending also under the fovea, and a small satellite lesion just temporal to the main deposit (Figure 2A). The main lesion had overlying orange pigment, also noticed on $\mathrm{AF}$ (Figure 2B), and subretinal fluid (Figure 2C). On B scan ultrasound, the larger of the two lesions measured $9 \mathrm{~mm}$ in diameter and $3.1 \mathrm{~mm}$ in elevation, it showed low-medium internal echogenicity and internal blood flow of $14 \mathrm{~cm} / \mathrm{sec}$ (Figure 2D). Given the medical history and intraocular findings of 2 discrete choroidal lesions, secondary spread to the choroid was suspected, despite the presence of orange pigment on the tumour surface and ultrasonographic characteristics. The patient was referred for external beam radiotherapy (EBRT) to the left eye but died a month later.

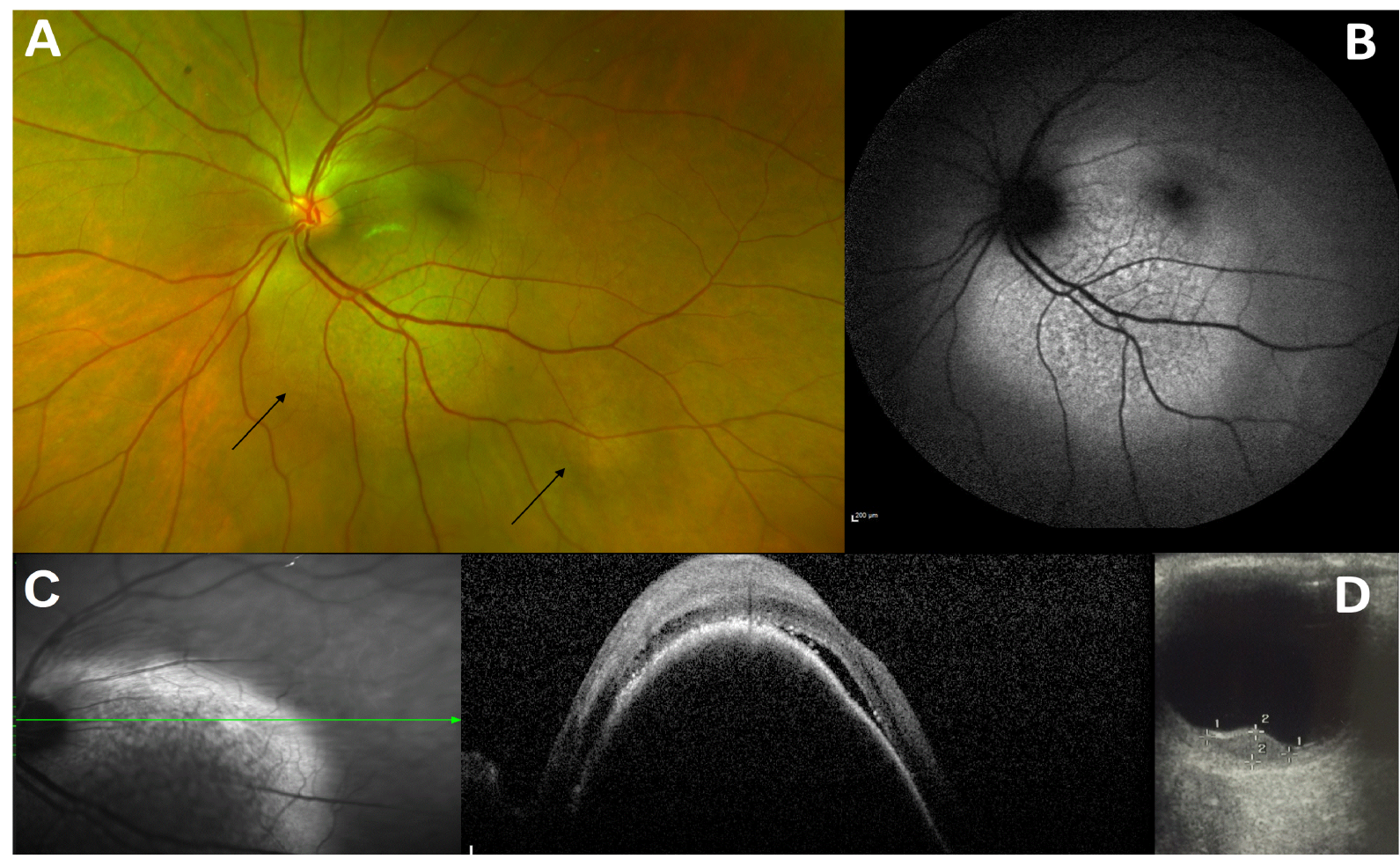

Figure 2. (A) Case 2 showing 2 posterior pole discrete choroidal lesion (arrows) with overlying orange pigment, (B) also seen as hyperfluorescent dots on autoflourescence, $(C)$ the choroidal lesions were also demonstrated on OCT, (D) and ultrasound.

\section{Case 3}

A 59-year old white woman with history of two months of blurred vision and a pale choroidal mass in the right eye was referred for evaluation and management in July 2015. Prior to her referral, a chest, abdomen and pelvis $\mathrm{CT}$ scan revealed a suspicious lung mass. She was a non-smoker but had a familial history of lung cancer with her aunt and grandfather affected. On examination, BCVA was $6 / 24$ in the right eye and $6 / 6$ in the left eye. A yellow amelanotic choroidal mass with overlying areas of orange lipofuscin pigment was seen in the superior part of the macula of the right eye (Figures 3A-3C). There was also overlying subretinal fluid (Figure 3D). On $B$ scan ultrasound (Figure 3E), the lesion had an irregular border with mixed medium-high internal echogenicity, a diameter of 8.8 $\mathrm{mm}$ and elevation of $3.2 \mathrm{~mm}$ with internal blood flow of 32 $\mathrm{cm} / \mathrm{sec}$. The mass demonstrated pinpoint late hyperfluorescence on FA (Figure 3F) and hyperfluorescence on ICG, with the absence of any signs suggestive of an inflammatory or vascular cause (Figure 3G). The differential diagnosis included a deposit from a suspicious, yet to be proved, lung cancer, versus primary choroidal melanoma. The patient was referred for a lung biopsy, which confirmed a diagnosis of large cell lung cancer, supporting an ocular diagnosis of choroidal metastasis with overlying orange pigment. The patient was treated with whole eye EBRT and systemic chemotherapy. On examination three months later, BCVA was 6/6 OU, the lesion OD appeared to have reduced in vertical height both on clinical appearance and on ultrasound (elevation of $1.5 \mathrm{~mm}$ ). 


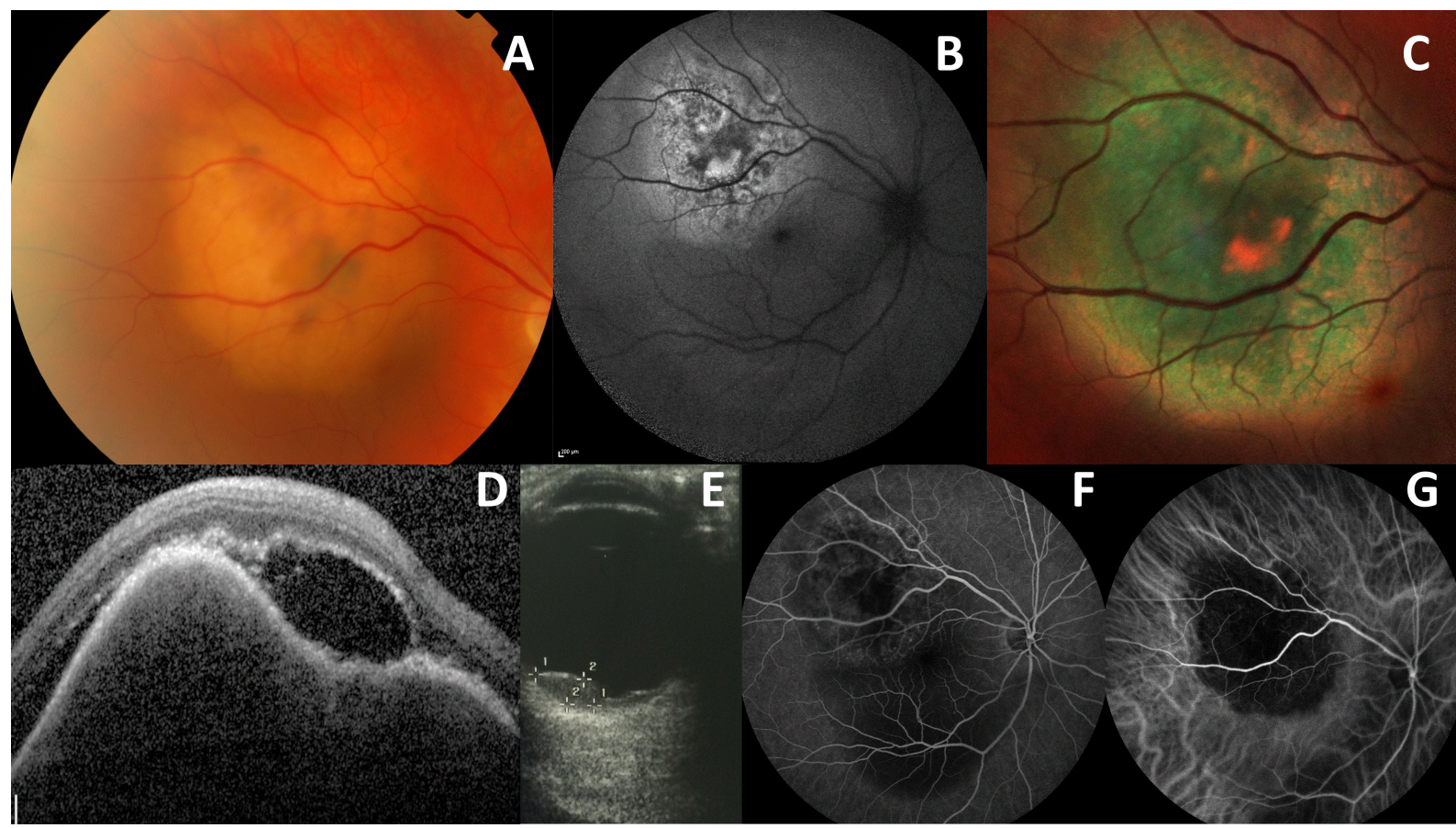

Figure 3. (A) Case 3 showing a pale posterior pole lesion (B) with overlying orange pigment also seen on autofluorescence scan, (C) MultiColor Scanning Laser Imaging, (D) Subretinal fluid is demonstrated on OCT, which also shows a "lumpy, bumpy" surface topography, (E) and the intraocular tumour on ultrasound, $(F)$ On fluorescein angiography, pinpoint late hyperfluorescence was demonstrated, $(G)$ and indocyanine green showed masking, without any features associated with granulomatous disease or a haemangioma.

\section{Discussion}

We herein report a case series of 3 patients, of whom 2 were known to have extraocular cancer and an the other with blameless medical history, diagnosed with choroidal metastases on clinical grounds despite the presence of orangecolor lipofuscin over the tumour surface. At times, differentiating an amelanotic choroidal melanoma from a choroidal metastasis is not straightforward. The diagnostic challenge is further heightened when no primary cancer site is known, estimated to occur in about third of cases $[11,12]$. Intraocular tissue biopsy can be used to make a diagnosis however biopsy of posterior pole tumours may pose risks to vision such as haemorrhage or retinal detachment. In our experience, many patients with disseminated metastatic disease are not well enough to undergo this procedure. In addition, fine needle aspiration biopsy may only yield a small tissue sample insufficient to reliably make a diagnosis. Systemic staging investigations may reveal the unknown primary tumour but more often there is a clear history of a previous malignancy.

In contrast to primary choroidal melanoma, choroidal metastases are usually pale, multifocal and can be bilateral in up to $48 \%$ of patients. One typical feature of choroidal metastasis is the association of a large serous retinal detachment, with often more subretinal fluid than found in a comparable sized melanoma [11-14]. Orange-color lipofuscin confirmed with AF imaging is considered a risk factor for future growth of a choroidal naevus. The new onset of lipofuscin on the surface of a choroidal naevus is highly suspicious for malignant transformation into choroidal melanoma [5-8]. For this reason, many ocular oncologists consider orange-color lipofuscin a hallmark of choroidal melanoma. While orange pigment is a relatively specific sign in this clinical scenario, it is not pathognomonic in helping to differentiate a choroidal metastasis from melanoma, as shown in our observations.

Shields et al. investigated 520 cases of choroidal metastasis [12], 14 of which had overlying orange pigment. However, they emphasized that it differs from the orange-color lipofuscin associated with choroidal melanoma in its geographic irregular appearance under the retina. We found it difficult, even in retrospect, to clinically associate the orange color in the cases reported in the present study to metastasis rather than melanoma. Moreover, AF scans, in which lipofuscin is seen as hyperfluorescent dots or clumps, suggested that the material is present, but was not useful in differentiating between the 2 choroidal tumour types. Recently, Riechardt et al. [15] reported a case of a 73 years old female patient with history of breast cancer and recent diagnosis of metastatic disease involving the lung, bones and choroid. The tumour showed orange pigment 2.5 years after initial presentation, when no orange pigment had been noted on previous examinations. Consistent with these studies, we are reporting 3 additional cases in which choroidal metastasis were found to have overlying orange lipofuscin pigment at diagnosis.

\section{Limitations}

Limitations of this study include its retrospective design and small cohort size. In addition, diagnoses of the choroidal metastases were clinical rather than tissue biopsy based. However, given the overall clinical picture, and patient outcome, it is highly likely that the choroidal tumours were a result of secondary spread. 
Citation: Masalha AN, Fabian ID, Cohen VML, et al. Choroidal metastasis presenting with tumour surface orange lipofuscin pigment. J Clin Ophthalmol 2019;3(1):132-136.

\section{Conclusion}

In conclusion, orange lipofuscin pigment commonly seen overlying choroidal melanoma, is also found overlying choroidal metastasis, hence does not on its own serve as a useful diagnostic tool to differentiate between the two. Other extraocular and ocular findings should be evaluated, and if in doubt, systemic workup should be performed to rule out an extraocular primary site.

\section{Statement of Ethics}

The study was approved by the Moorfields Eye Hospital institutional review board in concordance with the declaration of Helsinki (Reference number ROAD16/044).

\section{References}

1. Brunk UT, Terman A. Lipofuscin: Mechanisms of agerelated accumulation and influence on cell function. Free Radic Biol Med. 2002;33:611-619.

2. Terman A, Brunk UT. Molecules in focus lipofuscin. Int $\mathbf{J}$ Biochem Cell Biol. 2004;36:1400-1404.

3. Kennedy CJ, Rakoczy PE, Constable IJ. Lipofuscin of the retinal pigment epithelium: A review. Eye. 1995;9:763-771.

4. Shields CL, Shields JA, Kiratli H, et al. Risk factors for growth and metastasis of small choroidal melanocytic lesions. Ophthalmology. 1995;102(9):1351-1361.

5. Shields CL, Bianciotto C, Pirondini C, et al. Autofluorescence of orange pigment overlying small choroidal melanoma. Retina. 2007;27:1107-1111.

6. Shields CL, Bianciotto C, Pirondini $\mathrm{C}$, et al. Autofluorescence of choroidal melanoma in 51 cases. $\mathrm{Br} \mathrm{J}$ Ophthalmol. 2008;92:617-622.

7. Shields CL, Furuta M, Berman EL, et al. Choroidal nevus transformation into melanoma analysis of 2514 consecutive cases. Arch Ophthalmol. 2009;127(8): 981-987.

8. Garcia MD, Salomao DR, Marmorstein AD, et al. Histopathologic findings in the areas of orange pigment overlying choroidal melanoma s. Transl Vis Sci Technol. 2016;5(3):1-4.

9. Bloch RS, Gartner S: The incidence of ocular metastatic carcinoma. Arch Ophthalmol. 1971;85:67-65.

10. Krantz BA, Dave N, Komatsubara KM, et al. Uveal melanoma: Epidemiology, etiology, and treatment of primary disease. Clinical Ophthalmol. 2017;11:279-289.

11. Arepalli S, Kaliki S, Shields CL. Choroidal metastases: Origin, features, and therapy. Indian J Ophthalmol. 2015;63(2):122-127.

12. Shields CL, Shields JA, Gross NE, et al. Survey of 520 eyes with uveal metastases. Ophthalmology. 1997; 104:1265-1276.

13. Cohen VML. Ocular metastases. Eye. 2013;27:137-141.

14. Demirci H, Shields CL, Chao AN, et al. Uveal metastasis from breast cancer in 264 patients. Am J Ophthalmol. 2003;136:264-271.

15. Riechardt AI, Gundlach E, Joussen AM, et al. The development of orange pigment overlying choroidal metastasis. Ocul Oncol Pathol. 2015;1:93-97.

\section{*Correspondence to}

Ido Didi Fabian

Department of Ocular Oncology

Moorfields Eye Hospital

162 City Road, London EC1V 2PD, UK

Tel: $+44(0) 2072533411$

E-mail: didi@didifabian.com 